\title{
ANTI-ATHEROSCLEROTIC EFFECTS OF EURYCOMA LONGIFOLIA IN RATS FED ON HIGH-FAT DIET
}

\author{
Fakhria Al-Joufi ${ }^{1}$, Anil K. Saxena ${ }^{a}$, Imad M. Al-Ani ${ }^{1}$, Norlelawati A. Talib ${ }^{1}$, Rafidah H. Mokhtar ${ }^{2}$, Norsidah Ku-Zaifah $^{1}$
}

${ }^{1}$ Department of Basic Medical Sciences, Kulliyyah of Medicine, International Islamic University Malaysia, P. O. Box 141, 2571

Kuantan, Pahang, Malaysia. ${ }^{2}$ Faculty of Medicine, Universiti Sains Islam Malaysia, Nilai, Negeri Sembilan, Malaysia.

Introduction: Cardiovascular diseases (CVD) particularly atherosclerosis, have become a growing health problem, especially in developing countries. Hyperlipidemia is widely known as a dominant risk factor for the development of atherosclerosis.

Objective: This study was designed to investigate the effects of Eurycoma Longifolia (EL) on the testosterone level, its associated biochemical changes to the lipid profile and intima media thickness (IMT) in rats fed on high-fat diet.

Methodology: Twenty young, adult male Sprague-Dawley (SD) rats were housed for 12 weeks. After one week of acclimatization, they were randomly divided into four groups of 5 animals each and treated for 12 weeks as follows: Group ND was given only normal diet, group NDEL was given normal diet and EL extracts $(15 \mathrm{mg} / \mathrm{kg})$ dissolved in distilled water, group HFD was given only high fat diet and group HFDEL was given high fat diet and EL extracts $(15 \mathrm{mg} / \mathrm{kg})$.

Results: Rats which were treated with EL (NDEL and HFDEL) showed a significant increase $(\mathrm{p}<0.05)$ in the testosterone levels. There was a significant decrease $(p<0.05)$ in triglyceride $(T G)$ in the HFDEL group compared to the HFD group. The histological sections of aortas revealed a significant decrease $(p<0.05)$ in IMT in the HFDEL when compared with the HFD group. No histological changes were observed in the NDEL group compared with the ND group and there was no significant difference in IMT values between the NDEL and the ND groups.

Conclusion: These findings suggest that EL is a promising protective agent against atherosclerosis induced by HF diet.

\section{EFFECT OF FLAXSEED EXTRACT ON THE LIVER HISTOLOGICAL STRUCTURE IN STREPTOZOTOCIN INDUCED DIABETIC RATS}

Imad M. Al-Ani ${ }^{1}$, Ahmed N. Abired ${ }^{2}$, Basma E. Mustafa ${ }^{2}$, Emad N. Abdel Wahab ${ }^{1}$ and Marwan S. Azzubaidi ${ }^{2}$

${ }^{1}$ Department of Basic Medical Science, Kulliyyah of Medicine, International Islamic University Malaysia, Kuantan, Pahang, Malaysia. ${ }^{2}$ Department of Basic Medical Science, Kulliyyah of Dentistry, International Islamic University Malaysia, Kuantan, Pahang, Malaysia.

Introduction: Diabetes mellitus has become a serious public health issue all over the world. The management goal in diabetes is to keep blood glucose levels as close to normal as possible. Medications used to treat diabetes are usually associated with multiple adverse drug reactions. Recently, many traditional medications sourced from plants have become popular in the management of diabetes mellitus. Flaxseed has been used as a traditional medication for centuries and could potentially influence blood glucose levels.

Objective: This study aims to evaluate the hepatoprotective effects of flaxseed extract in streptozotocin (STZ)-induced diabetic rats.

Methodology: Diabetes mellitus was induced in Sprague-Dawley rats using a single injection of streptozotocin (60 $\mathrm{mg} / \mathrm{kg}$ intra-peritoneal). The rats were divided into five groups of 8 rats each. Group NC, normal control rats; Group $\mathrm{NF}$, normal rats treated with flaxseed extract (400 mg/kg); Group DC, diabetic control rats; Group DG, diabetic rats treated with glibenclamide $(0.6 \mathrm{mg} / \mathrm{kg})$; Group DF, diabetic rats treated with flaxseed extract $(400 \mathrm{mg} / \mathrm{kg})$; for 4 weeks.

Results: There were significant increases in relative liver weight, blood glucose levels in the DC group comparing to the NC group $(\mathrm{p}<0.05)$. The disturbance of these parameters was ameliorated in the DF and DG groups. Histological 